\title{
Development of a time-resolved white-light interference microscope for optical phase measurements during fs-laser material processing
}

\author{
Alexander Horn • Dirk Wortmann • Andreas Brand • \\ Ilya Mingareev
}

Received: 22 November 2009 / Accepted: 10 May 2010 / Published online: 12 June 2010

(C) Springer-Verlag 2010

\begin{abstract}
A modified Mach-Zehnder interferometer set-up combined with microscope objectives has been developed for the measurement of phase changes in the processed material sample, like modification and melting of glass. The white light is generated by focusing ultrafast laser radiation $\left(t_{\mathrm{p}}=80 \mathrm{fs}\right)$ in a sapphire crystal using a micro-lens array to minimize temporal and spatial fluctuations in the whitelight continuum. Lateral and coaxial pump-probe measurements of the phase changes during material processing are performed using two coupled ultrafast laser sources at different repetition rates $\left(f_{\text {rep }}=1 \mathrm{~Hz}-1 \mathrm{MHz}\right)$. The optical phase shift and therefore the refractive index of the material are calculated from the interference images using two approaches. The knowledge of the refractive index during the laser processing with a temporal resolution in the ps-range and a spatial resolution of several microns leads to a better understanding of the initial processes for the permanent material modifications.
\end{abstract}

\section{Introduction}

Ultrafast laser modification and ablation techniques are currently being adopted and developed further in many research

\footnotetext{
A. Horn ( $\varangle)$

Institut für Physikalische Chemie I, Georg-August Universität

Göttingen, Tammannstr. 6, 37077 Göttingen, Germany

e-mail: alexander.horn@mac.com

D. Wortmann · A. Brand · I. Mingareev

Lehrstuhl für Lasertechnik, RWTH Aachen University,

Steinbachstr. 15, 52074 Aachen, Germany

Present address:

I. Mingareev

Townes Laser Institute, University of Central Florida, 4000

Central Florida Blvd., Orlando, FL 32816, USA
}

and industrial branches. Recent advances in ultrafast laser sources [1] offer multi-MW peak pulse powers at multi$\mathrm{MHz}$ repetition rates. The ability to deposit energy in a short time period, before electronic relaxation takes place $(\approx 2$ ps [2]) is a key benefit of ultrafast laser radiation which enables a wide variety of applications.

In the trend for miniaturization of optical components and systems the joining of glass and writing of waveguides in the micrometer range, for example, are some of the challenges that have only been solved rudimentarily yet. A method for selective modifications in the volume of transparent dielectrics is the irradiation by ultra short laser pulses at repetition rates $>100 \mathrm{kHz}$ [3]. When focusing the ultrafast laser radiation into the volume of glass, the successive irradiation with femtosecond laser pulses serves as a heat point source inside the material. This leads to melting of material on the micrometer scale around the focus resulting in permanent changes of the microscopic structure and affecting properties like the refractive index [4]. A better understanding of such processes is of great importance for the technological implementation of waveguides writing and development of a waveguide-laser.

Optical interferometry is a well renowned technique to determine differences in optical thickness and thus refractive index. Phase detection by phase contrast [5] or monochromatic interferometry [6] with ultra short laser pulses is sporadically used in research. For time-resolved applications it has many shortcomings such as failing in the distinct determination of optical path differences larger than $\lambda / 2$. Whitelight interferometry uses broad spectrum illumination that offers unambiguous interference patterns. It has been technically implemented since the 1960s (e.g. Leica white-light interference microscopes by Horn) but has not been developed further for time-resolved applications. 


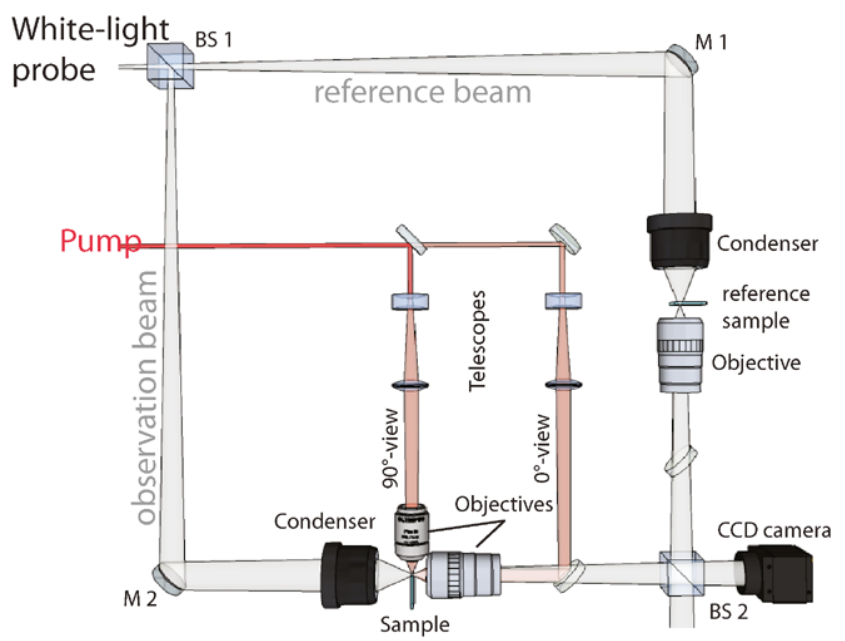

Fig. 1 Pump-probe white-light interference microscopy set-up

We describe the development of an ultrafast white-light interference microscope for observation of femtosecond laser pulse induced changes in refractive index. A pumpprobe imaging technique has been adopted to observe laserinduced phenomena on a time-scale up to eight nanoseconds while maintaining a temporal resolution of $\Delta t \approx 1 \mathrm{ps}$. To achieve this temporal resolution an ultrafast white-light source with an output pulse energy large enough for singleshot image generation $\left(E_{\mathrm{p}} \approx 10 \mu \mathrm{J}\right)$ has been developed. Commercially available ultrafast white-light sources such as based on photonic crystal fibers only provide output energies of $E_{\mathrm{p}} \ll 1 \mathrm{~nJ}$. We developed new algorithms to analyze the interference fringes, because common phase unwrapping methods are not applicable to single-shot interferograms.

Two in-situ experiments have been conducted to demonstrate the applicability and validate the conceived set-up.

\section{Interferometer set-up}

In order to gain the ability to conduct interferometric pumpprobe analysis a Mach-Zehnder interferometer set-up is conceived (Fig. 1). Like with any amplitude-splitting interferometer large spatial separations between the interferometer arms are achievable and offers enough space to shape and guide the interferometer beam paths to fit the demands of different pump-probe set-ups.

A beamsplitter (BS 1) is used to divide the amplitude of a light beam into an observation and a reference beam. One beam acts as a reference while the other passes through the object of interest where its phase gets shifted according to the sample's diffractive and refractive properties. In order to visualize this phase change as an interferogram the two beams have to be precisely superimposed and realigned via another beamsplitter (BS 2) and detected by a 8-bit color CCD camera (Arc4000c, Baumer Optronic,
$1300 \times 1030 \mathrm{Pixel}^{2}$ ). A small and controlled misalignment of one arm using one mirror (M 2) leads to an easily interpretable pattern of intensity (FRINGES). In the undisturbed case, meaning reference and probe beam do not undergo any phase alteration while being split apart, the intensity fringes will be evenly spaced and parallel as seen in Fig. 3 left. The difference between the ordinary Mach-Zehnder interferometer and a Mach-Zehnder interference microscope basically is that the beam path includes two sets of microscopes each consisting of a condenser and an objective, one for the reference and one for the observation arm (Fig. 1). A MachZehnder interference microscope requires two sets of microscope objectives and condensers that are matched as good as possible in terms of phase-distortion. Here two alignments between the pump and the probe beam are considered, coaxial with an observation angle of $\alpha=0^{\circ}$, and lateral alignment with an observation angle of $\alpha=90^{\circ}$ respectively. Coaxial alignment is realized via the interferometer objective as pump and probe objective at the same time and lateral alignment by using an additional objective for pumping. In order to take advantage of the benefits of both white-light interferometry and time-resolved pump-probe measurements a suitable supercontinuum source (SC) has been developed.

\section{Femtosecond white-light source}

Pump-probe experiments in the femtosecond regime require temporally constrained probe pulses in order to capture any dynamics on an image. To observe ultrafast laser-induced phenomena via white-light interferometry, a broadband light source is needed that can emit short pulses $\left(t_{\mathrm{p}} \ll n s\right)$ at an intensity large enough to sufficiently expose a CCD camera chip. Focusing femtosecond laser radiation in dielectrics generates a supercontinuum [7] possessing a high degree of spatial coherence.

The attempts to generate white-light pulses with sufficient energy to generate properly expose colored images with stable interference fringes by focusing the femtosecond laser radiation into a nonlinear medium with one lens failed. Either the white-light radiation exhibited enough illumination power but with small spatial coherence due to filamentation or the radiation featured sufficient coherence but at light intensity levels too small for proper image exposure.

We solved this by directing the femtosecond laser radiation through an array of 127 hexagonally shaped converging micro lenses, $f=18 \mathrm{~mm}, N A \approx 0.008$, forming an array of focuses accordingly (Fig. 2). When focused into a nonlinear medium such as a sapphire rod, each of the focuses will lead to self-focusing and cause filamentation with one filament only and supercontinuum generation of its own. Since the pulse energy is split between all micro lenses, the respective pulse energy per focus is decreased accordingly. 


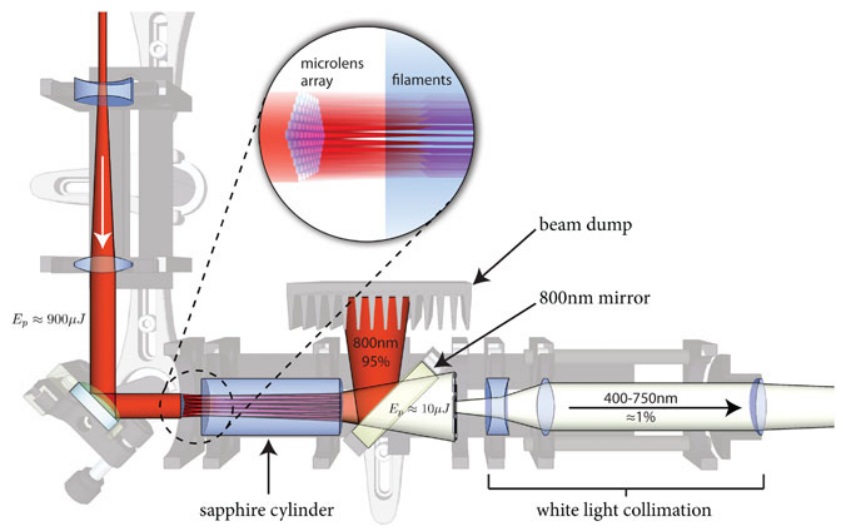

Fig. 2 Set-up for SC generation using micro-lens array

The separated broadband emissions overlap at the exit of the sapphire cylinder, since the spatial separation of each filament is the same as the micro-lens pitch $(300 \mu \mathrm{m})$. Using single-shot imaging results in sufficiently exposed interferograms (Fig. 3 left). The pulse-to-pulse repeatability of the interference pattern indicates a large degree of spatial coherence even though the micro-lens array and thus the array of focuses is evenly spread over several millimeters.

Cross-correlation is used to determine the pulse duration of the white light generated by the SC source. The total pulse duration of the broadband emission is determined to approximately $10 \mathrm{ps}$ for the used $50 \mathrm{~mm}$ long sapphire cylinder.

\section{Interferogram analysis}

Interferograms are images that contain intensity distributions (fringes) due to constructive and destructive interference. The fringe pattern represents the phase difference distribution of the probe and reference arm to analyze the degree and spatial distribution of the phase change. A common procedure is the phase-shifting algorithm [8]. Thereby four interferograms are captured sequentially in time at a path length difference of $\lambda / 4$ and allows for the unambiguous relative phase determination of each pixel [9]. Single interferograms can not be analyzed by this technique. In case of a pump-probe interferometer only one interferogram can be obtained at a time since it is not possible to readjust the set-up before the affected area has changed its appearance and phase properties. When limited to one interferogram, the phase information has to be determined by the fringe position and shape. A 2D Fourier-transform approach [6] can not be used to reconstruct the phase in this case though, because it is only applicable for radiation with large coherence lengths since a large number of fringe orders is needed for the evaluation.

The first approach we describe is a method to calculate quantitative phase changes from interferograms given
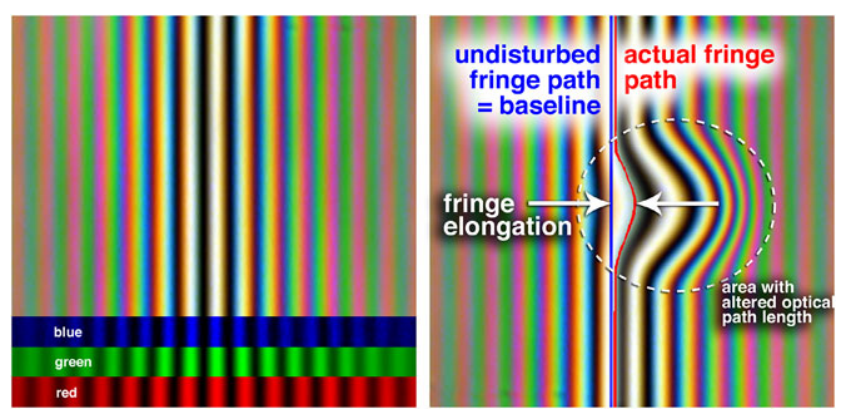

Fig. 3 The interferometer is set up to produce straight vertical fringes (left). In this case the optical path difference (OPD) is zero at the center maximum

by measuring the fringe displacements. The procedure has been implemented into a MATLAB program for quick phase analysis of batches of interferograms. For all interference measurements, the interferometer is set up to produce measurably equidistant and straight fringes before any measurement (Fig. 3, left). This fringe configuration then represents a homogeneous optical path length distribution.Any modification of the refractive index $(\Delta n)$ with thickness $d$ along the beam path then varies the optical path length $O P L=$ $n \cdot d=\int n(s) d s$ accordingly. This gives the optical path difference $O P D=O P L_{2}-O P L_{1}=\Delta n \cdot d=\int \Delta n(s) d s$. As a result, the fringe pattern, or interference order at the position $x$ will resemble the original pattern at $x+O P D$ (Fig. 3). The fringe elongation $x$ is defined as the perpendicular distance of a fringe segment of an interferogram to the respective fringe segment of the undisturbed case, where $O P D=0$ across the image (Fig. 3 right). The undisturbed fringe can be derived from the interferogram itself. Since the interferometer is aligned to produce straight fringes and any modifications causing an $O P D$ are limited to the inner part of the interferograms, a linear interpolation between the top and bottom pixels generates the undisturbed BASELINE for this fringe. For every fringe this method provides displacement values along its path.

Our second approach to determine the phase change is to compare each row of pixels of the interferogram with the analog row of a generated undisturbed image. For example two comparative rows of an interferogram are evaluated resulting in intensity distribution with a red line representing the measurement interferogram and a green line representing the respective undisturbed image (Fig. 4 top). The algorithm compares the intensity values of both rows. The gradient of the intensity of the surrounding points is evaluated in order to calculate possible fringe elongations via intensity comparison throughout the row resulting in a final image (Fig. 4 bottom).

A coaxial pump alignment is used to induce melt tracks in BK7 glass with the sample being moved perpendicularly through the focus area. The laser source for the pump radiation is an IMRA fiber laser (FCPA $\mu$-Jewel D-1000) with 


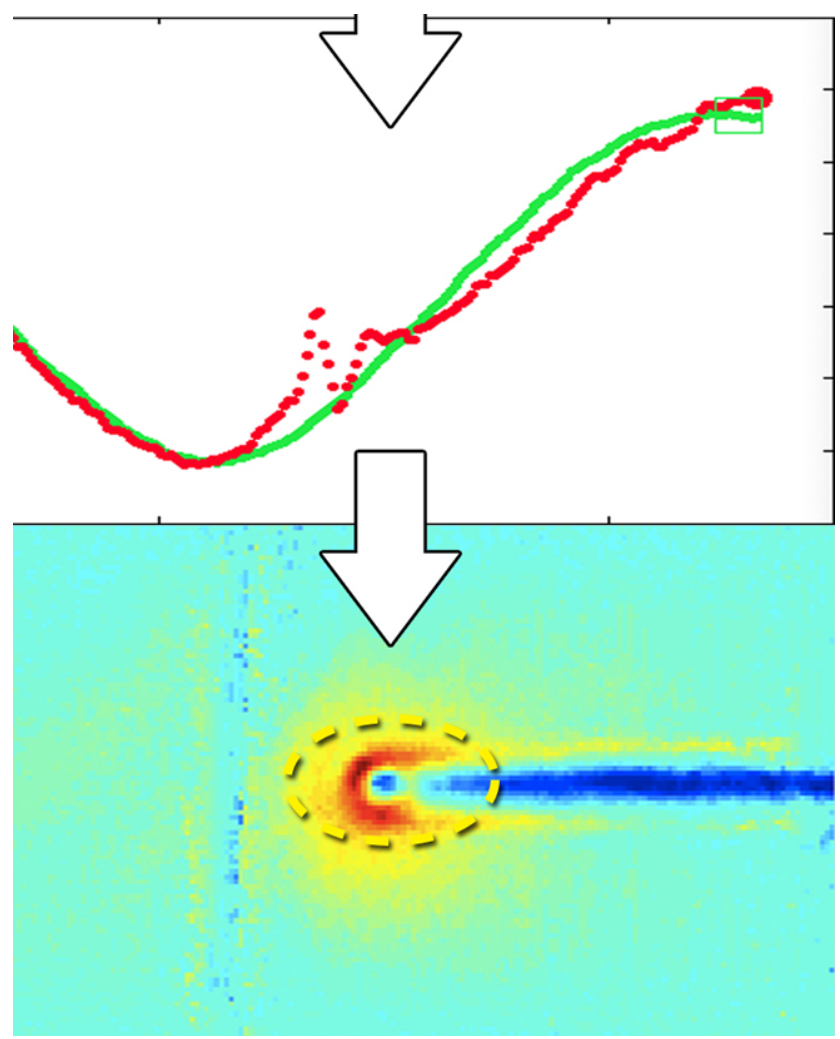

Fig. 4 (Top) Intensity distribution of interferogram and undisturbed image. (Bottom) Final image after pixelwise phase algorithm

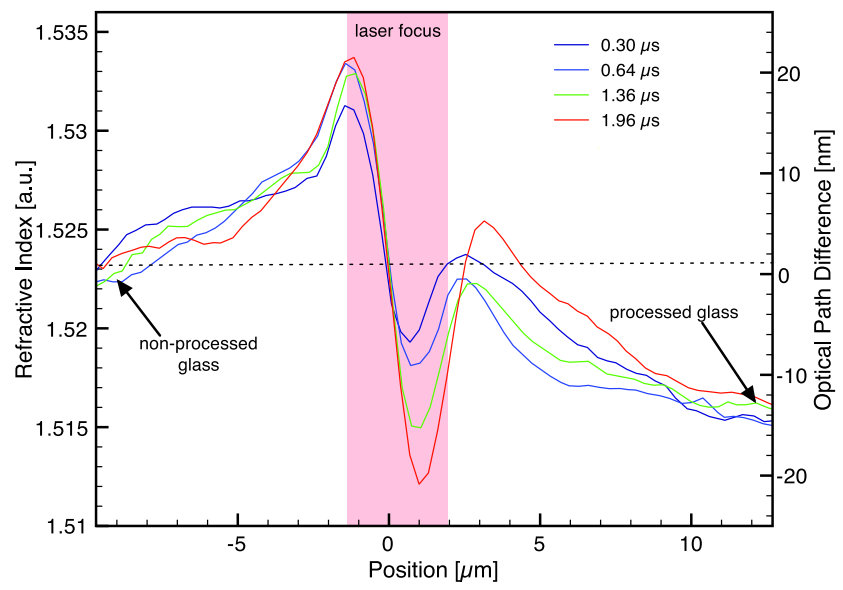

Fig. 5 Transient refractive index and $O P D$ changes coaxially along a melt track

pulse duration $t_{\mathrm{p}}=500 \mathrm{fs}$ and a spectral bandwidth of $\Delta \lambda \approx$ $2 \mathrm{~nm}$. The repetition rate was adjusted to $f_{\text {rep }}=200 \mathrm{kHz}$, the translation speed to $v=26 \mu \mathrm{m} / \mathrm{s}$, and the average output power to $P_{\mathrm{av}}=135 \mathrm{~mW}$. To investigate the temporal changes of the $O P D$ interferograms were taken at four delays $\tau$ with all laser parameters kept constant (Fig. 5). The highlighted area at position $x=0$ indicates the effective area of the laser focus. The decrease of the $O P D$ and refractive

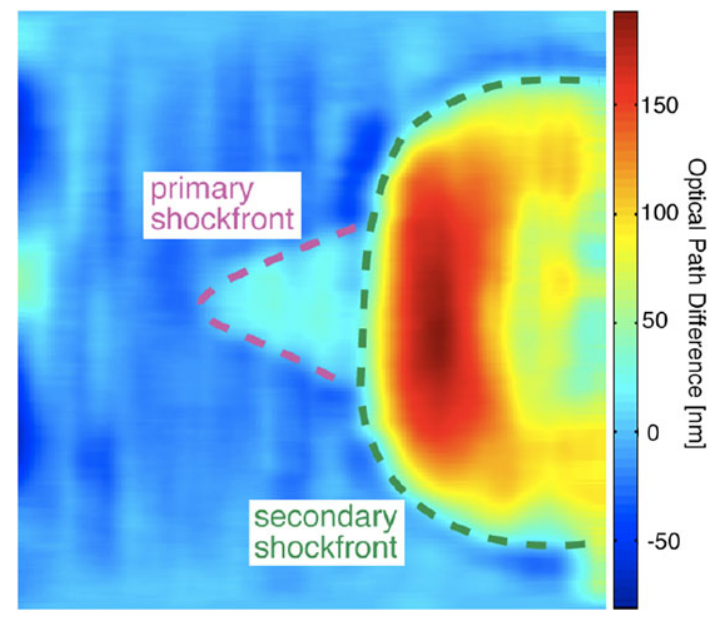

Fig. 6 Calculated phase distribution $4.6 \mathrm{~ns}$ after single- pulse ablation of aluminum ( $f_{\text {rep }}=1 \mathrm{~Hz}, \lambda=800 \mathrm{~nm}$, and $E_{\mathrm{p}}=150 \mu \mathrm{J}$ )

index there can be attributed to the generation of free electrons [10]. Right of the focus area a region with an relatively increased $O P D$ is detected. The increase is caused by compressed and molten glass which was heated by electronic relaxation. Further to the right the $O P D$ decreases for all delays until reaching a constant level which indicates that heat has dissipated. The unprocessed glass is optically thicker than the processed one which causes a positive $O P D$ and refractive index change. On the left border of the diagram the glass is not affected yet.

A plasma plume after single-pulse ablation of aluminum is detected using the lateral alignment for the pump source. Radiation of a regenerative amplifier (Thales CONCERTO) at $f_{\text {rep }}=1 \mathrm{~Hz}, \lambda=800 \mathrm{~nm}$, and $E_{\mathrm{p}}=150 \mu \mathrm{J}$ is focused on the metal surface using a $20 \times$ microscope objective (NA 0.3). The phase distribution of an ablation plume is detected $4.6 \mathrm{~ns}$ after irradiation revealing two shockfronts (Fig. 6).

In summary we have presented an innovative diagnostic tool for time-resolved detection of laser-induced optical phase changes. This Mach-Zehnder interference microscope features a pump-probe module for coaxial an lateral detection using a speckle-free ultrafast white-light supercontinuum source with $\Delta t \approx 1 \mathrm{ps}$ pulse duration. The dynamics of heating and melting of glass has been detected and the phase distribution of a plasma plume after femtosecond laser radiation excitation of aluminum has been detected.

\section{References}

1. P. Russbueldt, T. Mans, G. Rotarius, J. Weitenberg, H.D. Hoffmann, R. Poprawe, Opt. Express 17(15), 12230-12245 (2009)

2. I.H. Chowdhury, A.Q. Wu, X. Xu, A.M. Weiner, Appl. Phys. A 81, 1627-1632 (2005)

3. S.M. Eaton, H. Zhang, J. Li M.L. Ng, W.J. Chen, S. Ho, P.R. Herman, Opt. Express 16, 9443-9458 (2008) 
4. K.M. Davis, K. Miura, N. Sugimoto, K. Hirao, Opt. Lett. 21, 1729 (1996)

5. A. Mermillod-Blondin, J. Bonse, A. Rosenfeld, I.V. Hertel, Yu.P. Meshcheryakov, N.M. Bulgakova, E. Audouard, R. Stoian, Appl. Phys. Lett. 94, 041911 (2009)

6. V.V. Temnov, K. Sokolowski-Tinten, P. Zhou, A. El-Khamhawy, D. von der Linde, Phys. Rev. Lett. 97, 237403 (2006)

7. A. Brodeur, S.L. Chin, J. Opt. Soc. Am. B 16(4), 637-650 (1999)
8. H. Beyer, Theorie und Praxis der Interferenzmikroskopie (Akademische Verlagsgesellschaft Geest und Portig, Leipzig, 1974)

9. P. de Groot, X.C. de Lega, J. Kramer, M. Turzhitsky, Appl. Opt. 41, 4571-4578 (2002)

10. A. Horn, I. Mingareev, J. Gottmann, A. Werth, U. Brenk, Meas. Sci. Technol. 19, 015302 (2008) 